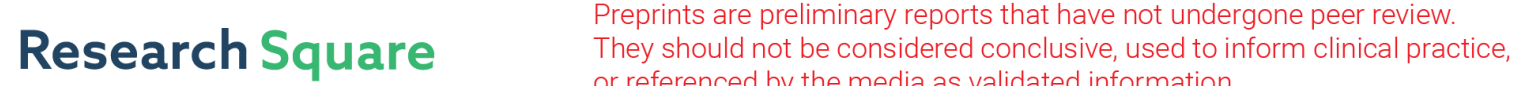 or referenced by the media as validated information. \\ Node and Edge Eigenvector Centrality for Hypergraphs
}

Francesco Tudisco ( $\nabla$ francesco.tudisco@gssi.it)

Gran Sasso Science Institute

Desmond Higham

University of Edinburgh

Article

Keywords: node and edge eigenvector centrality, pairwise interactions, nonlinear Perron-Frobenius theory

Posted Date: January 26th, 2021

DOl: https://doi.org/10.21203/rs.3.rs-148524/v1

License: (9) This work is licensed under a Creative Commons Attribution 4.0 International License. Read Full License

Version of Record: A version of this preprint was published at Communications Physics on September 2nd, 2021. See the published version at https://doi.org/10.1038/s42005-021-00704-2. 


\title{
Node ANd EdGe EIGENVECTOR CENTRALITy FOR HYPERGRAPHS
}

\author{
Francesco Tudisco \\ School of Mathematics \\ Gran Sasso Science Institute \\ 67100, L'Aquila (Italy) \\ francesco.tudisco@gssi. it
}

\author{
Desmond J. Higham \\ School of Mathematics \\ University of Edinburgh \\ EH93FD, Edinburgh (UK) \\ d.j.higham@ed.ac.uk
}

\begin{abstract}
Network scientists have shown that there is great value in studying pairwise interactions between components in a system. From a linear algebra point of view, this involves defining and evaluating functions of the associated adjacency matrix. Recent work indicates that there are further benefits from accounting directly for higher order interactions, notably through a hypergraph representation where an edge may involve multiple nodes. Building on these ideas, we motivate, define and analyze a class of spectral centrality measures for identifying important nodes and hyperedges in hypergraphs, generalizing existing network science concepts. By exploiting the latest developments in nonlinear Perron-Frobenius theory, we show how the resulting constrained nonlinear eigenvalue problems have unique solutions that can be computed efficiently via a nonlinear power method iteration. We illustrate the measures on realistic data sets.
\end{abstract}

\section{Motivation}

The study of pairwise interactions has led to a vast range of useful concepts and tools in network science [15, 26]. Several recent studies have developed extensions that account for higher order interactions [6, 16, 31]. Of course, the appropriate higher order representation is dependent on the research problem being addressed. For example, as discussed in [31], in studying coauthorship data set we could pose three distinct questions:

1. Have two given authors ever contributed simultaneously to a multi-authored paper? A simple undirected graph records such pairwise interactions.

2. Has a given set of authors ever contributed simultaneously to a multi-authored paper? Because any subset of these authors must also have contributed simultaneously to a multi-authored paper, we may use a simplicial complex to record these interactions. This structure incorporates downward closure: any subset of nodes within a simplex also forms a simplex.

3. Has a given set of authors formed the complete coauthorship list on a paper? In this case, a hypergraph is appropriate, with the set of authors forming a hyperedge. Any proper subset of those authors will not appear as a hyperedge unless they form the complete coauthorship list on some other paper.

In this work, we look at eigenvector-based centrality measures, thereby focusing on spectral algorithms [34]. These have been widely used to assign levels of importance to individual nodes. Our overall aim is to develop a centrality measure for (nonuniform, nonregular) hypergraphs that can assign importance both to nodes and hyperedges. We work in terms of the incidence matrix, and hence the material immediately transfers to the case of simplicial complexes, where all subsets of each hyperedge happen to be present. 
Eigenvector centrality for graphs assign scores to nodes in terms of the Perron eigenvector of the adjacency matrix of the graph [9, 15]. A standard approach when dealing with hypergraphs is to use graph-based algorithms on the clique-expanded graph of the hypergraph [1]. In this case, we can assign centrality scores to the nodes of the hypergraph by looking at the centrality of the nodes in its cliqueexpanded graph.

Another relatively well-established idea to represent and work with hypergraphs relies on the use of a higher-order tensors [4, 7]. This approach requires a uniform hypergraph, which can be fully described by an adjacency tensor, and can be used to define the centrality scores in terms of the Perron eigenvector of the adjacency tensor.

The clique-expansion matrix approach is a form of flattening which essentially corresponds to an additive model: the importance of the nodes on each hyperedge is summed into a linear (possibly weighted) combination. Instead, the tensor-eigenvector approach is a multiplicative model: the importances of the nodes on each hyperedge are multiplied. While the two models coincide on standard graphs (as each hyperedge contains exactly two nodes in that case), these two models are intrinsically different on uniform hypergraphs with larger edges.

We review both the clique-expansion and the tensor-based centrality models in detail in Sections 3.2 and 3.3 .

In this work we define an eigenvector model for node and edge centralities on general hypergraphs based on the choice of four nonlinear functions. We show that both the clique-expansion and, for uniform hypergraphs, the tensor-based eigenvector centralities are particular cases of the proposed model. Thus, our approach allows us to extend tensor-eigenvector centrality models to general hypergraphs. More precisely, the main contributions of this work are:

- the constrained nonlinear eigenvalue problem (2)

- in the special case of a 2-uniform hypergraph this leads to a HITS-type iteration for networks that simultaneously assigns centrality to nodes and edges,

- for general hypergraphs the resulting iteration assigns centrality to nodes and hyperedges,

- Theorem 4.1; existence and uniqueness theory for (2),

- Theorem 4.2. convergence theory for a nonlinear power method iteration designed for (2),

- Section 5. computational experiments.

\section{Network Centrality}

In this section we consider an unweighted, undirected, graph $G=(V, E)$ with node set $V=\{1, \ldots, n\}$, edge set $E=\left\{e_{1}, \ldots, e_{m}\right\}$ and binary adjacency matrix $A \in \mathbb{R}^{n \times n}$.

\subsection{Eigenvector Centrality for Nodes}

Eigenvector centrality assigns a measure of importance, $x_{i}>0$, to node $i$ in such a way that the importance of node $i$ is linearly proportional to the sum of the importances of its neighbours. This relationship may be written

$$
A \boldsymbol{x}=\lambda \boldsymbol{x}, \quad \boldsymbol{x}>0, \quad \text { for some } \lambda>0 .
$$

Thanks to the Perron-Frobenius theorem, this matrix eigenvector problem admits a unique solution $\boldsymbol{x}^{*}$ if $A$ is irreducible (that is, the graph is connected) [15]. In this case, $\boldsymbol{x}^{*}$ can be computed to arbitrary precision via the power method if $A$ is primitive (that is, the graph is aperiodic), see, for example, [33]. Eigenvector centrality was popularized in the social network science community [9], but the idea can traced back to the 19th Century [29].

\subsection{Eigenvector Centrality for Nodes and Edges}

Over the years, a large amount of work has been devoted to the definition of centrality models able to capture different network properties and thus provide an importance score to the nodes of a graph. 
However, much less work has focused on models and methods for quantifying centrality of edges. In addition to being of interest in its own right, quantifying edge importance has natural applications in a number of important tasks, including link detection, edge prediction and matrix completion [21, 23, 30].

An eigenvector centrality for edges can be developed by considering the line graph and its adjacency matrix $A^{(e)}$ [11]. In this setting, $A^{(e)} \in \mathbb{R}^{m \times m}$ has $A_{e_{1}, e_{2}}^{(e)} \neq 0$ if and only if $e_{1} \in E$ and $e_{2} \in E$ share at least one node. The centrality of the edges can thus be defined via the Perron eigenvector $A^{(e)} \boldsymbol{y}=\lambda \boldsymbol{y}$, as in (1).

Another somewhat natural model for edge centrality can be induced by a given node centrality $\boldsymbol{x}$ : assign to each edge $e$ the score $y_{e}=\sum_{i \in e} x_{i}$ obtained by looking at the nodes the edge connects. However, if $\boldsymbol{x}$ does not depend on $\boldsymbol{y}$, this approach is intrinsically local, as the importance of the edges does not take into account the structure of connections in the graph (similarly to degree centrality for nodes). A more informative centrality score for nodes and edges can be designed by requiring a mutual reinforcement property where edges inherit importance from the nodes they connect and, vice-versa, nodes inherit importance from the edges they participate in. We describe this idea in detail in the next section. Since the resulting model extends essentially unchanged to the higher-order setting where edges contain arbitrary number of nodes, we will present this idea in the framework of a hypergraph.

\section{Hypergraph Centrality}

We now consider a general hypergraph $H=(V, E)$ where $V=\{1, \ldots, n\}$ is the set of nodes and $E=\left\{e_{1}, \ldots, e_{m}\right\}$ now denotes the set of hyperedges [27]. Note that in our setting every node can belong to an arbitrary number of hyperedges, i.e., we allow hereditary hypergraphs [10], which can also be used to model a simplicial complex structure. We let $B$ denote the $n \times m$ incidence matrix of $H$, defined as follows: the rows of $B$ correspond to nodes while its columns correspond to hyperedges and we have $B_{i, e}=1$ if node $i$ takes part in hyperedge $e$, that is,

$$
B_{i, e}= \begin{cases}1 & i \in e \\ 0 & \text { otherwise. }\end{cases}
$$

In many situations we have access to external node and edge weights in the form of weight functions $\nu$ : $\mathbb{R}^{V} \rightarrow \mathbb{R}_{+}$and $w: \mathbb{R}^{E} \rightarrow \mathbb{R}_{+}$. For example, if the hypergraph data represents grocery goods (the nodes) and the list of items purchased by each customer in one visit to a supermarket (the hyperedges), then $\nu(i)$ can be an indicator of the price of item $i$, while $w(e)$ may correspond to the profit the supermarket has made with the list of items in $e$. We use two diagonal matrices $N$ and $W$ to take into account for these weights, defined by $N=\operatorname{Diag}(\nu(1), \ldots, \nu(n))$ and $W=\operatorname{Diag}\left(w\left(e_{1}\right), \ldots, w\left(e_{m}\right)\right)$.

Note that a hypergraph where all edges have exactly two nodes is a standard graph. In that case, we have $B W B^{\top}=A+D$ where $A$ is the adjacency matrix of the graph and $D=\operatorname{Diag}\left(d_{1}, \ldots, d_{n}\right)$ is the digonal matrix of the weighted node degrees $d_{i}=\sum_{e \in E} w(e) B_{i, e}=(B W \mathbb{1})_{i}$. Similarly, for a general hypergraph $H$, we have $B W B^{\top}=A_{H}+D_{H}$ where $A_{H}$ and $D_{H}$ are the adjacency and degree matrices of the clique-expansion graph $G_{H}=\left(V, E_{H}\right)$ associated with $H$. The clique-expansion graph is a graph on the same vertex set of $H$, obtained by adding a weighted clique connecting all nodes in each hyperedge of $H$. More precisely, given $H=(V, E)$, we have

$$
\left(A_{H}\right)_{i j}=\sum_{e: i, j \in e} w(e), \quad\left(A_{H}\right)_{i j}=0 .
$$

Thus, $i j \in E_{H}$ if and only if $i \neq j$ participates in at least one hyperedge of $H$. Similarly, the degree matrix $D_{H}=\operatorname{Diag}\left(d_{1}, \ldots, d_{n}\right)$ is the diagonal matrix whose diagonal entries are the weighted degrees of the nodes in the hypergraph, i.e. $d_{i}=\sum_{e: i \in e} w(e)=(B W \mathbb{1})_{i}$.

\subsection{Node and edge nonlinear hypergraph centrality}

We describe here a spectral (thus mutually reinforcing) model for node and edge centralities of hypergraphs. Suppose $H=(V, E)$ is given with $|V|=n$ and $|E|=m$, and let $\boldsymbol{x} \in \mathbb{R}^{n}, \boldsymbol{y} \in \mathbb{R}^{m}$ be 
nonnegative vectors whose entries will provide centrality scores for the nodes and hyperedges of $H$, respectively. We would like the importance $y_{e}$ for an edge $e \in E$ to be a nonnegative number proportional to a function of the importances of the nodes in $e$, for example $y_{e} \propto \sum_{i \in e} \nu(i) x_{i}$. Similarly, we require that the centrality $x_{i}$ of node $i \in V$ is a nonnegative number proportional to a function of the importances of the edges it participates in, for example $x_{i} \propto \sum_{e: i \in e} w(e) y_{e}$. As the centralities $x_{i}$ and $y_{e}$ are all nonnegative, these sums coincide with the weighted $\ell^{1}$ norm of specific sets of centrality scores. Thus, we can generalize this idea by considering the weighted $\ell^{p}$ norm of node and edge importances. This leads to

$$
x_{i} \propto\left(\sum_{e: i \in e} w(e) y_{e}^{p}\right)^{1 / p}, \quad y_{e} \propto\left(\sum_{i \in e} \nu(i) x_{i}^{q}\right)^{1 / q},
$$

for some $p, q \geq 1$. More generally, we can consider four functions $f, g, \varphi, \psi: \mathbb{R}_{+} \rightarrow \mathbb{R}_{+}$of the nonnegative real line $\mathbb{R}_{+}$and require that

$$
x_{i} \propto g\left(\sum_{e: i \in e} w(e) f\left(y_{e}\right)\right), \quad y_{e} \propto \psi\left(\sum_{i \in e} \nu(i) \varphi\left(x_{i}\right)\right) .
$$

If we extend real functions on vectors by defining them as mappings that act in a componentwise fashion, the previous relations can be compactly written as the following constrained nonlinear equations

$$
\left\{\begin{array}{l}
\lambda \boldsymbol{x}=g(B W f(\boldsymbol{y})) \\
\mu \boldsymbol{y}=\psi\left(B^{\top} N \varphi(\boldsymbol{x})\right)
\end{array} \quad \boldsymbol{x}, \boldsymbol{y}>0, \quad \lambda, \mu>0 .\right.
$$

If $f, g, \psi$ and $\varphi$ are all identity functions, then (2) boils down to a linear system of equations which is structurally reminiscent of the HITS centrality algorithm for directed graphs, [5, 24]. HITS computes two different node centralities: a $h u b$ centrality, which is proportional to the authority score of neighboring nodes, and at the same time, authority centrality, which is proportional to the hub score of neighboring nodes. Similarly, (2) with $f=g=\varphi=\psi=$ id defines two centralities, but in this case they relate to nodes and hyperedges: the importance of a node is proportional to the sum of the importances of the hyperedges it belongs to and, vice-versa, the importancesof a hyperdge is proportional to the sum of the importances of the nodes it involves.

As for HITS centrality, when $f=g=\varphi=\psi=$ id and we have no edge nor node weights (i.e. $W, N$ are identity matrices), then $\boldsymbol{x}, \boldsymbol{y}$ in (2) are the left and right singular vectors of a certain matrix, in this case $B$, and the matrix Perron-Frobenius theory tells us that if the bipartite graph with adjacency matrix $\left(\begin{array}{cc}0 & B \\ B^{\top} & 0\end{array}\right)$ is connected, then (2) has a unique solution. Instead, when either $f, g, \varphi$ or $\psi$ is not linear, even the most basic question of existence of a solution to (2) is not straightforward. However, for homogeneous functions $f, g, \varphi$ and $\psi$, the nonlinear Perron-Frobenius theory for multihomogeneous operators [19] allows us to give guarantees on existence, uniqueness and computability for the nonlinear singular-vector centrality model in (2).

Before addressing these issues, we investigate the system (2) further, showing how it includes some previously proposed eigenvector centrality models as special cases, and offers additional useful flexibility.

\subsection{The linear case: eigenvector centrality for graph and line graph}

When $H$ is a standard simple and unweighted graph $H=G=(V, E)$, with binary adjacency matrix $A$, it is easy to verify that $B B^{\top}=A+D$, where $D$ is the diagonal matrix of the node degrees. Moreover, $B^{\top} B=A^{(e)}+\Delta$, where $A^{(e)}$ is the adjacency matrix of the line graph of $G$ and $\Delta=\operatorname{Diag}\left(\delta_{1}, \ldots, \delta_{m}\right)$ is a diagonal matrix whose diagonal entries count the number of nodes each edge contains. In this case, each edge has exactly two nodes, so $\Delta=2 I$. The corresponding identities hold if we allow weights on the nodes and on the edges of $G$, namely $B W B^{\top}=A+D$, where now $A$ and $D$ are the weighted adjacency and degree matrix of $G$, and $B^{\top} N B=A^{(e)}+\Delta$ with

$$
\left(A^{(e)}\right)_{e_{1}, e_{2}}= \begin{cases}\nu(i) & e_{1} \neq e_{2} \text { and they share the node } i \text { in } G \\ 0 & \text { otherwise }\end{cases}
$$

and $\delta_{e}=\sum_{i \in e} \nu(i)$. 
It follows that, when $H$ is a graph, the node-edge eigenvector model (2) for the linear case $f=g=\varphi=$ $\psi=\mathrm{id}$ is strongly related to the standard eigenvector centrality applied to $G$ and its line graph. In fact, by using the two identities $\lambda \boldsymbol{x}=B W \boldsymbol{y}$ and $\mu \boldsymbol{y}=B^{\top} N \boldsymbol{x}$ we obtain

$$
\left\{\begin{array}{l}
\widetilde{\lambda} \boldsymbol{x}=B W B^{\top} N \boldsymbol{x}=(A+D) N \boldsymbol{x} \\
\widetilde{\lambda} \boldsymbol{y}=B^{\top} N B W \boldsymbol{y}=\left(A^{(e)}+\Delta\right) W \boldsymbol{y}
\end{array}\right.
$$

with $\widetilde{\lambda}=\lambda \mu$. Thus, $\boldsymbol{x}$ and $\boldsymbol{y}$ are the Perron eigenvectors of diagonally perturbed adjacency matrices of the graph and the line graph.

A similar connection holds for the general hypergraph case. In that case, the node-edge eigenvector model (2) for the linear choices $f=g=\varphi=\psi=$ id is tightly connected to the eigenvector centrality of the clique-expansion graph of $H$ its line graph. Precisely we have

$$
B W B^{\top}=A_{H}+D_{H} \quad \text { and } \quad B^{\top} N B=A_{H}^{(e)}+\Delta_{H},
$$

where $A_{H}^{(e)}$ and $\Delta_{H}$ are the adjacency and degree matrix of the line graph of $G_{H}$, as defined in (3).

\subsection{Tensor-based eigenvector centrality for uniform hypergraphs}

A hypergraph is said to be $k$-uniform if $|e|=k$ for all $e \in E$. Thus, a 2-uniform hypergraph is a graph in the standard sense. The concept of eigenvector centrality has been extended to the case of $k$-uniform hypergraphs with $k>2$ by means of the hypergraph adjacency tensor [7]. As every hyperedge contains exactly $k$ nodes, we can associate to $H$ a tensor $\mathcal{A}$ with $k$ indices $\mathcal{A}_{i_{1}, \ldots, i_{k}}$ such that $\mathcal{A}_{i_{1}, \ldots, i_{k}}=w(e)$ if the hyperedge $e=\left\{i_{1}, \ldots, i_{k}\right\}$ belongs to $E$, and $\mathcal{A}_{i_{1}, \ldots, i_{k}}=0$ otherwise. Clearly, $\mathcal{A}$ coincides with the adjacency matrix of the graph when $k=2$. Different notions of tensor eigenvectors are available in the literature (see e.g., [13, 20]). In particular, for $p>0$, a $\ell^{p}$ eigenvector for $\mathcal{A}$ is a vector $\boldsymbol{x}$ such that

$$
\sum_{i_{2}, \ldots, i_{k}} \mathcal{A}_{i_{1}, i_{2}, \ldots, i_{k}} x_{i_{2}} x_{i_{3}} \cdots x_{i_{k}}=\lambda x_{i_{1}}^{p} .
$$

The special cases $p=1$ and $p=k-1$ correspond to so-called $Z$ - and $H$-eigenvectors for $\mathcal{A}$. Note that both $Z$ - and $H$-eigenvectors boil down to standard matrix eigenvectors when $k=2$. However, when $k>2$ things are significantly different. In particular, the eigenvector centrality defined by (4) is no longer linear when $k>2$, in the sense that taking a linear combination of eigenvectors does not automatically produce an eigenvector.

This nonlinearity makes the analysis and the computation of solutions to (4) more challenging than standard eigenvector centralities for graphs (i.e. $k=2$ ). However, it has been observed in, e.g., [18, 32, 20] that nonlinear eigenvector equations of the form (4) admit a unique solution that can be computed to an arbitrary precision if the tensor $\mathcal{A}$ is not too sparse and if the exponent $p$ satisfies certain assumptions. In particular, for a large range of values of $p$ all these properties hold with almost no requirement on the connectivity of the underlying hypergraph [13]. From this point of view, the nonlinearity yields a remarkable advantage rather than a disadvantage.

In the next subsection we highlight an intriguing connection between the tensor-based eigenvector centrality (4) and the model proposed in (2), which is based solely on the incidence matrix of the hypergraph.

\subsubsection{Incidence matrix formulation of tensor eigenvectors}

Let $H$ be a $k$-uniform hypergraph. As observed above, when $k=2$ we have $B W B^{\top}=A+D$, where $A$ and $D$ are the adjacency and degree matrices of the graph $H$, respectively. Thus, for $p=2$ we can easily rewrite the eigenvector centrality equation (4) in terms of the incidence matrix, as (4) coincides with $A \boldsymbol{x}=\lambda \boldsymbol{x}$ and we have $A \boldsymbol{x}=\left(B W B^{\top}-D\right) \boldsymbol{x}=\lambda \boldsymbol{x}$. If the vector $\boldsymbol{x}$ is entrywise positive, we can add a nonlinear transformation in the eigenvector equation to obtain a similar relation for any $k \geq 2$ and any $p \geq 1$. More precisely, we have

Theorem 3.1. Let $H$ be a $k$-uniform hypergraph with $\nu(i)=1$ for all $i \in V$. If $\boldsymbol{x}$ is a positive solution of (2) with $f(\boldsymbol{x})=\boldsymbol{x}, g(\boldsymbol{x})=\boldsymbol{x}^{1 /(p+1)}, \psi(\boldsymbol{x})=e^{\boldsymbol{x}}$ and $\varphi(\boldsymbol{x})=\ln (\boldsymbol{x})$, then $\boldsymbol{x}$ is an eigenvector centrality solution of the tensor eigenvalue problem (4). 
Proof. First note that with $f=$ id and $g(\boldsymbol{x})=\boldsymbol{x}^{1 /(p+1)}$ and $N=I$, from (2) we get $\lambda^{p+1} \boldsymbol{x}^{p+1}=B W \boldsymbol{y}$ and $\mu \boldsymbol{y}=\psi\left(B^{\top} \varphi(\boldsymbol{x})\right)$ which together imply that $\alpha \boldsymbol{x}^{p+1}=B W \psi\left(B^{\top} \varphi(\boldsymbol{x})\right)$ for some $\alpha>0$. Now, as every edge $e$ contains exactly $k$ nodes, we can write $e=\left\{i_{1}, \ldots, i_{k}\right\}$, yielding

$$
\psi\left(B^{\top} \varphi(\boldsymbol{x})\right)_{e}=\exp \left(\sum_{j \in e} \ln \left(x_{j}\right)\right)=x_{i_{1}} \cdots x_{i_{k}} .
$$

Furthermore, for any $i_{1} \in V$ and any $\boldsymbol{y} \in \mathbb{R}^{m}$ we have $(B W \boldsymbol{y})_{i_{1}}=\sum_{e: i_{1} \in e} w(e) y_{e}$. Thus, if $\mathcal{A}$ is the adjacency tensor of $H$ (defined by $\mathcal{A}_{i_{1}, \ldots, i_{k}}=w(e)$ if $e=\left\{i_{1}, \ldots, i_{k}\right\} \in E$ and $\mathcal{A}_{i_{1}, \ldots, i_{k}}=0$ otherwise) we get

$$
\left[B W \psi\left(B^{\top} \varphi(\boldsymbol{x})\right)\right]_{i_{1}}=\sum_{e: i_{1} \in e} w(e) \psi\left(B^{\top} \varphi(\boldsymbol{x})\right)_{e}=\sum_{i_{2}, \ldots, i_{k}} \mathcal{A}_{i_{1}, \ldots, i_{k}} x_{i_{1}} \cdots x_{i_{k}} .
$$

This shows that if $\boldsymbol{x}$ solves (2) then $\boldsymbol{x}$ must be such that

$$
\sum_{i_{2}, \ldots, i_{k}} \mathcal{A}_{i_{1}, i_{2}, \ldots, i_{k}} x_{i_{1}} x_{i_{2}} \cdots x_{i_{k}}=\alpha x_{i_{1}}^{p+1} .
$$

Finally, as $\boldsymbol{x}$ is positive, we can divide the previous identity by $x_{i_{1}}$ which reveals that $\boldsymbol{x}$ solves the tensor eigenvalue problem (4).

\section{Existence, uniqueness and computation of nonlinear hypergraph centralities}

In this section we discuss existence, uniqueness, positivity, maximality and computation of the node and edge hypergraph centrality defined by the general nonlinear singular value problem (2). Analogously to the linear case, these properties will follow directly from the nonlinear Perron-Frobenius theorem for multihomogeneous mappings [19], which extends the classical Perron-Frobenius theory for nonnegative matrices to a much broader class of nonlinear nonnegative operators.

To this end, we recall that a function $\varphi$ is said to be $\alpha$-homogeneous if $\varphi(\lambda \boldsymbol{u})=\lambda^{\alpha} \varphi(\boldsymbol{u})$ for all $\lambda \geq 0$. In this case we say that $\alpha$ is the homogeneity degree of $\varphi$. Furthermore, we say that $\varphi$ is order preserving if $\varphi(\boldsymbol{v}) \geq \varphi(\boldsymbol{u})$ for all $\boldsymbol{v} \geq \boldsymbol{u}$; whereas we say that $\varphi$ is positive if $\varphi(\boldsymbol{v})>0$ for all $\boldsymbol{v}>0$. We have

Theorem 4.1. Let $f, g, \varphi, \psi$ be order preserving and homogeneous of degrees $\alpha, \beta, \gamma, \delta$, respectively. Consider the $2 \times 2$ matrix

$$
M=\left(\begin{array}{cc}
0 & |\alpha \beta| \\
|\gamma \delta| & 0
\end{array}\right)
$$

and let $\rho(M)$ denote its spectral radius. If either

P1. $\rho(M)<1$, or

P2. $\rho(M)=1 ; f, g, \varphi, \psi$ are differentiable and positive maps; the bipartite graph with adjacency matrix $\left(\begin{array}{cc}0 & B W \\ B^{\top} N & 0\end{array}\right)$ is connected

then there exist unique $\boldsymbol{x}, \boldsymbol{y}>0$ (up to scaling) and unique $\lambda, \mu>0$ solution of (2).

Proof. The proof follows directly from the Perron-Frobenius theorem for multihomogeneous mappings [19]. Below we highlight the main steps. Let $F: \mathbb{R}^{n} \times \mathbb{R}^{m} \rightarrow \mathbb{R}^{n} \times \mathbb{R}^{m}$ be the mapping

$$
F(\boldsymbol{x}, \boldsymbol{y})=\left(g(B W f(\boldsymbol{y})), \psi\left(B^{\top} N \varphi(\boldsymbol{x})\right)\right) .
$$

A simple computation shows that $F$ is order-preserving and multihomogeneous with homogeneity matrix $M$ and a solution of (2) coincides with the multihomogeneous eigenvalue equation $F(\boldsymbol{x}, \boldsymbol{y})=(\lambda \boldsymbol{x}, \mu \boldsymbol{y})$. Thus, the thesis follows directly from [19, Thm. 3.1] under assumption P1. Assume now P2 holds. Since the mappings $f, g, \varphi, \psi$ act entry-wise, are homogeneous and order preserving, we have that the graph $\mathcal{G}(F)$, as per [19, Def. 5.1], coincides with the bipartite graph with adjacency matrix $\left(\begin{array}{c}0 \\ B^{\top}\end{array}{ }_{0}^{B W}\right)$. Thus, by [19, Thm. 5.2] there exist positive solutions to (2). Now let $(\boldsymbol{u}, \boldsymbol{v})>0$ be any such solution and let $D F(\boldsymbol{u}, \boldsymbol{v})$ be the Jacobian matrix of $F$ evaluated at $(\boldsymbol{u}, \boldsymbol{v})$. Since $f, g, \varphi, \psi$ are positive and homoge-

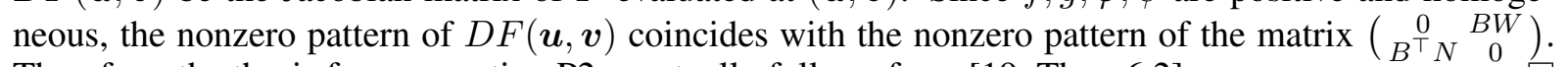
Therefore, the thesis for assumption P2 eventually follows from [19, Thm. 6.2]. 


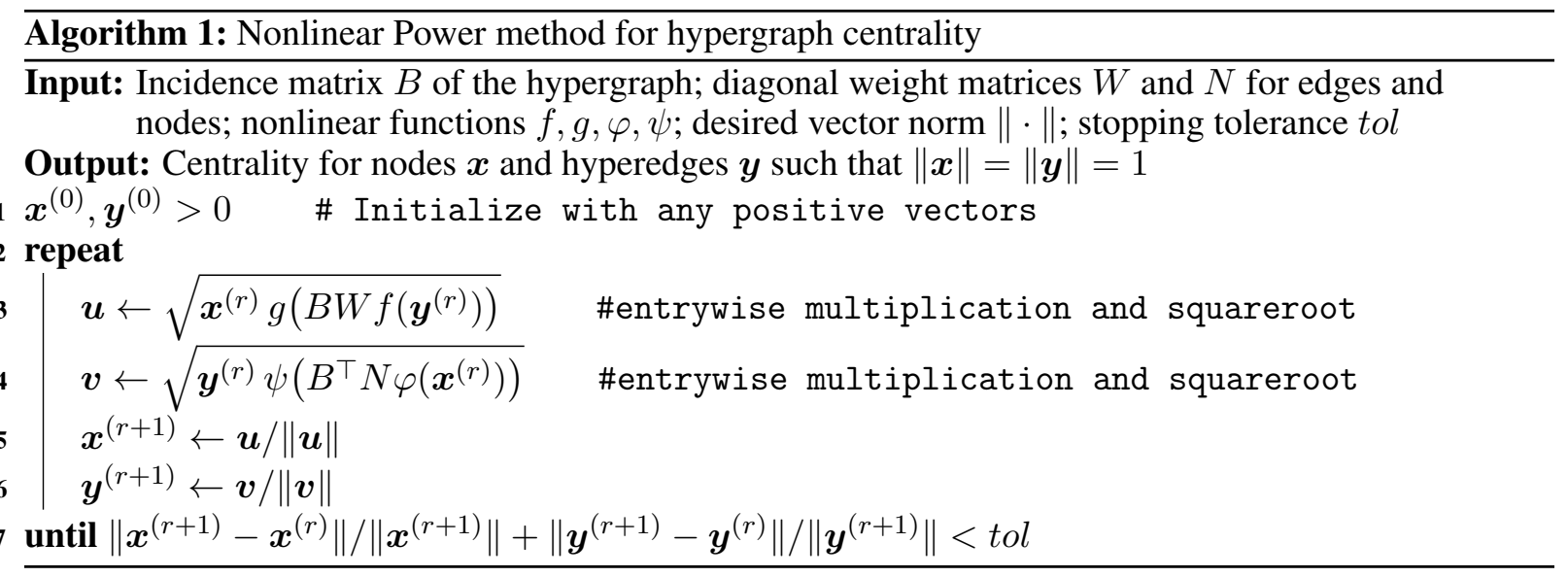

By analogy with the linear case, we refer to the positive solutions of (2) defining the hypergraph centralities as nonlinear Perron singular vectors.

On top of existence and uniqueness guarantees, the matrix Perron-Frobenius theorem provides us with the convergence of the so-called power method, a very powerful tool for computing the Perron singular vectors. In the case of nonnegative matrices, however, one needs to require the bipartite graph of $A=$ $\left(\begin{array}{cc}{ }^{0} & B W \\ B^{\top} N & 0\end{array}\right)$ to be aperiodic (i.e. the matrix $A$ is primitive) in order to ensure the convergence of the power method for an arbitrary choice of the starting point, as connectedness alone is not enough. As an example, consider the $2 \times 2$ matrix $A=\left(\begin{array}{ll}0 & 1 \\ 1 & 0\end{array}\right)$ which acts on vectors by swapping the first and the second coordinates. The graph of $A$ is connected but not aperiodic and, indeed, the sequence $\boldsymbol{x}^{(r+1)}=A \boldsymbol{x}^{(r)}$ does not converge in general (it converges only if $\boldsymbol{x}^{(0)}$ has constant entries).

Much like the matrix case, one can compute the nonlinear Perron singular vectors in (2) via what we call a nonlinear Power method, described in Algorithm 1. However, similarly to the nonlinear eigenvalue problem for tensors [20], the nonlinear power method converges under significantly milder conditions than its more common linear counter part. In particular, no aperiodicity assumption is required and, depending on the homogeneity degree of the nonlinear functions, global convergence may be ensured even for disconnected graphs. The following theorem describes the convergence of Algorithm 1 to the solution of (2).

Theorem 4.2. Under the assumptions in Theorem 4.1. let $\boldsymbol{x}^{(r)}, \boldsymbol{y}^{(r)}$ be the sequences generated by Algorithm 1$]$ If either $P 1$ or $P 2$ holds, then $\boldsymbol{x}^{(r)}$ and $\boldsymbol{y}^{(r)}$ converge to the unique positive solutions $\boldsymbol{x}^{*}, \boldsymbol{y}^{*}$ of (2) such that $\left\|\boldsymbol{x}^{*}\right\|=\left\|\boldsymbol{y}^{*}\right\|=1$. Moreover, if P1 holds, then the convergence is linear, i.e.:

$$
\left\|\boldsymbol{x}^{(r)}-\boldsymbol{x}^{*}\right\|+\left\|\boldsymbol{y}^{(r)}-\boldsymbol{y}^{*}\right\|=O\left(\rho(M)^{r}\right) .
$$

Proof. This proof follows almost directly from the case of tensor eigenvectors, discussed in [20, Thm. 3.3]. We tailor the main ideas of that argument to our hypergraph eigenvalue problem (2). Consider the mapping $G: \mathbb{R}^{n} \times \mathbb{R}^{m} \rightarrow \mathbb{R}^{n} \times \mathbb{R}^{m}$, defined by

$$
G(\boldsymbol{x}, \boldsymbol{y})=\left(\sqrt{\boldsymbol{x} g(B W f(\boldsymbol{y}))}, \sqrt{\boldsymbol{y} \psi\left(B^{\top} N \varphi(\boldsymbol{x})\right)}\right)
$$

where all the operations are intended entrywise. It is not difficult to verify that $G$ is order-preserving and multihomogeneous, with homogeneity matrix $\widetilde{M}=\frac{1}{2}(M+I)$ and that, if $F$ is defined as in the proof of Theorem 4.1, then $(\boldsymbol{x}, \boldsymbol{y})$ is such that $F(\boldsymbol{x}, \boldsymbol{y})=(\lambda \boldsymbol{x}, \mu \boldsymbol{y})$, for positive $\lambda$ and $\mu$, if and only if $G(\boldsymbol{x}, \boldsymbol{y})=(\widetilde{\lambda} \boldsymbol{x}, \widetilde{\mu} \boldsymbol{y})$, with $\widetilde{\lambda}, \widetilde{\mu}>0$. Moreover, a direct computation shows that the Jacobian matrices $D F(\boldsymbol{x}, \boldsymbol{y})$ and $D G(\boldsymbol{x}, \boldsymbol{y})$ of $F$ and $G$, respectively, are such that

$$
D G(\boldsymbol{x}, \boldsymbol{y})=\frac{1}{2} \operatorname{Diag}(G(\boldsymbol{x}, \boldsymbol{y}))^{-1 / 2}(\operatorname{Diag}(F(\boldsymbol{x}, \boldsymbol{y}))+\operatorname{Diag}((\boldsymbol{x}, \boldsymbol{y})) D F(\boldsymbol{x}, \boldsymbol{y}))
$$


where $\operatorname{Diag}(\boldsymbol{v})$ is the diagonal matrix with diagonal entries the elements of $\boldsymbol{v}$. As observed in the proof of Theorem 4.1, for a positive vector $(\boldsymbol{x}, \boldsymbol{y})$, the matrix $D F(\boldsymbol{x}, \boldsymbol{y})$ is irreducible. Thus, from (5), $D G(\boldsymbol{x}, \boldsymbol{y})$ is primitive and the thesis eventually follows from [19, Thm. 7.1].

\section{Experiments}

\subsection{Three singular vector model test cases}

In this section we compare the behaviour of three node-edge eigenvector centrality models which correspond to three choices of the functions $f, g, \varphi, \psi$ in (2), as described below:

Linear. This centrality corresponds to the linear choice $f=g=\varphi=\psi=$ id which, as discussed in Section 3.2, essentially corresponds to the standard eigenvector centrality applied to the graph and the line graph obtained by clique-expanding the input hypergraph.

Log-exp. This centrality corresponds to the choices $f=\mathrm{id}, \varphi(x)=\ln (x), \psi(x)=\exp (x)$ and $g(x)=\sqrt{x}$. As discussed in Section 3.3.1 this choice generalizes the ZEC eigenvector centrality proposed in [7] to the case of nonuniform hypergraphs. In fact, when the hypergraph is uniform, we have already observed that the node centrality defined via (2) with this choice of $f, g, \varphi, \psi$ boils down to a $Z$-eigenvector of the adjacency tensor of the hypergraph. Similarly, for a general hypergraph, from (2) we get

$$
\mu y_{e}=\psi\left(\sum_{j \in e} \nu(j) \varphi\left(x_{j}\right)\right)=\exp \left(\sum_{j \in e} \nu(j) \ln \left(x_{j}\right)\right)=\prod_{j \in e} x_{j}^{\nu(j)} .
$$

Thus, if $\boldsymbol{x}, \boldsymbol{y}$ are nonnegative vectors satisfying (2) there exists a positive $\widetilde{\lambda}$ such that

$$
\widetilde{\lambda} x_{i}^{2}=x_{i}^{\nu(i)} \sum_{e: i \in e} w(e) \prod_{j \in e \backslash\{i\}} x_{j}^{\nu(j)} .
$$

Note in particular that, when the input hypergraph has binary node weights, i.e., $\nu(i)=1$ for all $i \in V$, (6) corresponds to a nonuniform hypergraph version of the ZEC tensor eigenvector centrality for uniform hypergraphs, precisely we have

$$
\tilde{\lambda} x_{i}=\sum_{e: i \in e} w(e) \prod_{j \in e \backslash\{i\}} x_{j}^{\nu(j)} .
$$

Max. The function $\mu_{\alpha}(\boldsymbol{v})=\left(v_{1}^{\alpha}+\cdots+v_{m}^{\alpha}\right)^{1 / \alpha}$ is a type of 'softmax': when $\alpha \rightarrow \infty$ it converges to $\max (\boldsymbol{v})=\max \left\{v_{1}, \ldots, v_{m}\right\}$. More precisely, we have

$$
\max (\boldsymbol{v}) \leq\left(v_{1}^{\alpha}+\cdots+v_{m}^{\alpha}\right)^{1 / \alpha} \leq m^{1 / \alpha} \max (\boldsymbol{v}),
$$

thus, already for $\alpha=10$ we have $\mu_{\alpha}(\boldsymbol{v}) \approx \max (\boldsymbol{v})$. Our 'max centrality' corresponds to the choice of nonlinear mappings: $f=g=\mathrm{id}, \varphi(x)=x^{\alpha}$ and $\psi(x)=\varphi^{-1}(x)=x^{1 / \alpha}$, for $\alpha=10$.

Notice that, with this choice of $\alpha$, the max node centrality $x_{i}$ is large when $i$ is part of at least one important edge. In fact, from (2) we have

$$
x_{i} \approx \max \left\{y_{e}: e \supset i\right\} .
$$

\subsection{Illustrative example: hypergraph sunflower}

A sunflower is a hypergraph whose hyperedges all have one common intersection in one single node, called the core. Let $u \in V$ be that intersection. Also let $r$ be the number of petals (the hyperedges) each containing $\left|e_{i}\right|$ nodes, for $i=1, \ldots, r$. By definition $u \in e_{i}$ for all $i$. Further, a node $v \in e_{i}$ and $v \in e_{j}$ for $i \neq j$ if and only if $v=u$. 

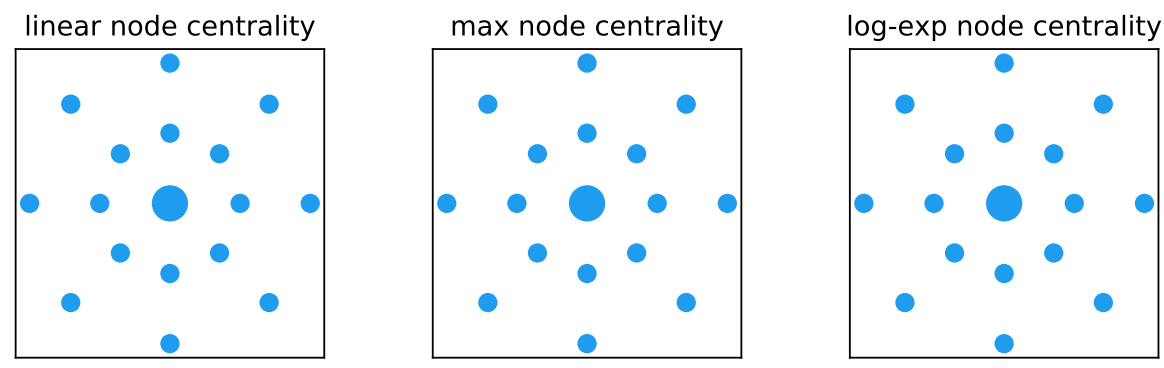

(a) Uniform sunflower with eight petals each containing three nodes
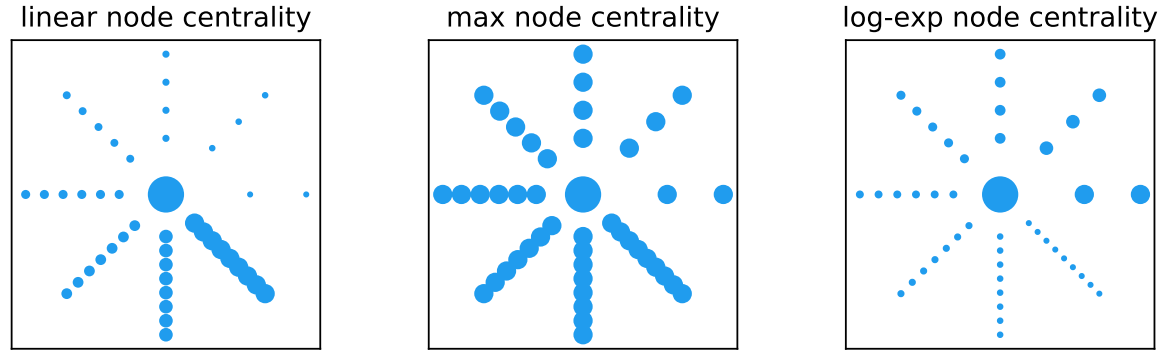

(b) General hypergraph sunflower with eight petals containing $3,4, \ldots, 10$ nodes, respectively.

Figure 1: Node centrality scores for the three models in Sec. 5.1 on the two example hypergraph sunflowers. Dots represent the hypergraph nodes and their size is proportional to their centrality value.

\section{Uniform sunflower}

If $\left|e_{i}\right|=k+1$ for all $i$ we say that the hypergraph is a uniform sunflower. The tensor eigenvector centrality of a uniform sunflower is studied for example in [7]. In that case we can assume that all the hyperedges have the same centrality score and that the same holds for all the nodes, besides the core, which is assigned a specific value.

Assuming no weights on nodes or hyperedges, by symmetry we may impose the constraints $x_{v_{i}}=x_{v}$ for all $v_{i} \neq u$ and $y_{e}=y$ for all $e \in E$ in (2) to obtain

$$
x_{v} \propto g(f(y)), \quad x_{u} \propto g(r f(y)), \quad y \propto \psi\left(\varphi\left(x_{u}\right)+k \varphi\left(x_{v}\right)\right) .
$$

So, for example, with the choices of Theorem 3.1 we get $x_{u} / x_{v}=g(r)=r^{1 /(p+1)}$ which coincides with the value computed in [7], for the two choices $p=1$ and $p=m-1$ (there named ZEC and HEC, respectively). More generally, if $g$ is homogeneous of degree $\beta$ we have

$$
\frac{x_{u}}{x_{v}} \propto r^{\beta} .
$$

This shows that the node centrality assignment in the case of a uniform sunflower hypergraph only depends on the homogeneity degree of $g$ and, in particular, when $\beta \rightarrow 0$ all the centralities tend to coincide, while $x_{u}>x_{v}$ for all $\beta>0$, confirming and extending the observation in [7] for the setting of uniform hypergraph centralities based on tensor eigenvectors. Figure 1a illustrates this behaviour on an example uniform sunflower hypergraph with eight petals $(r=8)$ each having three nodes $(k=3)$. The figure shows the nodes of the hypergraph with a blue dot whose size is proportional to its centrality value computed according to the three singular vector hypergraph centrality models defined in Sec. 5.1. The value of $\beta$ for these three centralities is 1 for both the 'max' and the 'linear' centrality', and 1/2 for 'log-exp' centrality'. Thus, all the three models assign essentially the same centrality score: the core node $u$ has strictly larger centrality, while all other nodes have same centrality score.

\section{Generic sunflower}

The situation is different for the case of a nonuniform hypergraph sunflower where we have $r$ petals each containing an arbitrary number of nodes. The computational results in Figure $1 \mathrm{~b}$ indicate that the three 


\begin{tabular}{ccc}
\hline Linear & Max & Log-exp \\
\hline Calculus & Calculus & Linear algebra \\
Real analysis & Real analysis & Probability \\
Integration & Linear algebra & Calculus \\
Sequences and series & Probability & Real analysis \\
Limits & Abstract algebra & Complex analysis \\
Analysis & Integration & Algebra precalculus \\
Derivatives & Sequences and series & General topology \\
Linear algebra & Matrices & Differential equations \\
Multivariable calculus & General topology & Combinatorics \\
Definite integrals & Combinatorics & Geometry \\
\hline
\end{tabular}

Table 1: Top ten nodes in the math stackexchange co-tags hypergraph, according to the three centrality models in Section 5.1

models in Sec. 5.1 capture significantly different centrality properties: All three models recognize the core node as the most central one, however while the 'linear' model favours nodes that belong to large hyperedges, the multiplicative 'log-exp' model behaves in the opposite way assigning a larger centrality score to nodes being part of small hyperedges. Finally, the 'max' model behaves like in the uniform case, assigning the same centrality value to all the nodes in the petals (core node excluded). It would be of interest to pursue these differences analytically and hence gain further insights into the effect of $f, g, \varphi$ and $\psi$.

\subsection{Real-world hypergraph data}

In this section we analyze the proposed nonlinear node-edge hypergraph centrality model on two realworld datasets. The code used to compute the results of this section is written in julia and is available at https://github.com/ftudisco/node-edge-hypergraph-centrality.

Walmart trips This is a transactional dataset that consists of a hypergraph describing customer trips at Walmart: hyperedges are sets of co-purchased products at Walmart, as released as part of the Kaggle competition in [22]. The hypergraph data is taken from [3]. Products are assigned a label which points to one of ten broad departments in which each product appears on walmart.com (e.g., "Clothing, Shoes, and Accessories"). There is also an additional "Other" class. The hyperedge weights are given by the number of times that a particular set of products appears in the list of co-purchased items. We summarize relevant statistics for this dataset in the list below:

- number of nodes: 88,860; number of hyperedges: 65,979 ;

- maximum edge weight: 679; mean / variance of edge weights: 1.06 / 15.24;

- maximum edge size: 25 ; mean edge size 6.86 .

Math stackexchange co-tags This is a temporal higher-order network dataset from [8]. Here we use the whole dataset ignoring the temporal component. The resulting dataset is a sequence of simplices where each simplex is a set of nodes. In this dataset, nodes are tags and simplices are the sets of tags applied to questions on math. stackexchange.com. We represent the dataset as a hypergraph with one hyperedge for each simplex. As before, the weight of each hyperedge is an integer number counting how many times that hyperedge appears in the data. Some basic statistics of this dataset are:

- number of nodes: 1,629; number of hyperedges: 170,476;

- maximum edge weight: 16,230; mean / variance of edge weights: 4.82 / 9,430.71;

- maximum edge size: 5; mean edge size: 3.48 .

In Figure 2 we scatter-plot the node and edge centrality obtained with these three models on the Walmart trip and the Math Stack-exchange co-tag hypergraphs. We normalize the values of each centrality vector by dividing them entry-wise by their largest entry (so that their largest value is scaled to one). The figure compares the three centrality models in a pair-wise fashion and shows no apparent linear correlation 
Math stackexchange co-trips node centrality
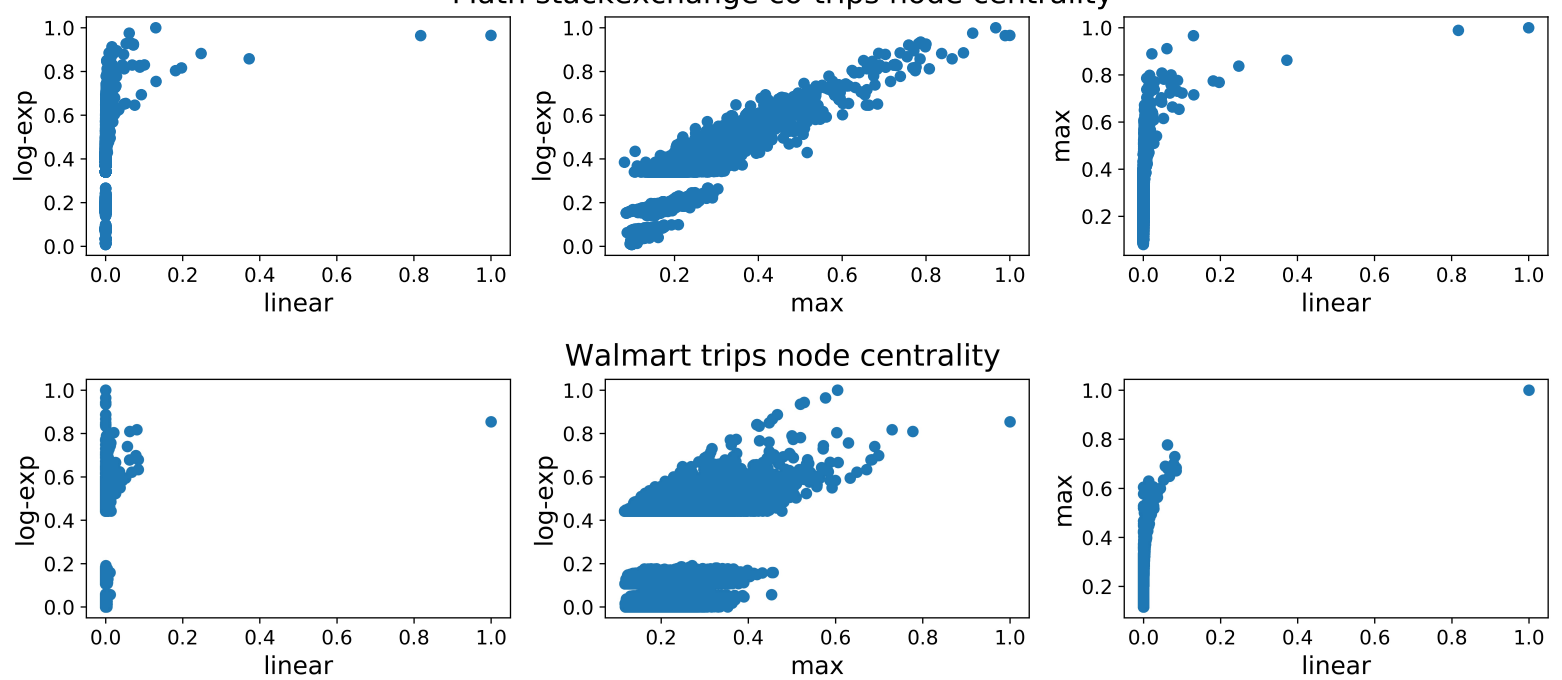

Walmart trips node centrality
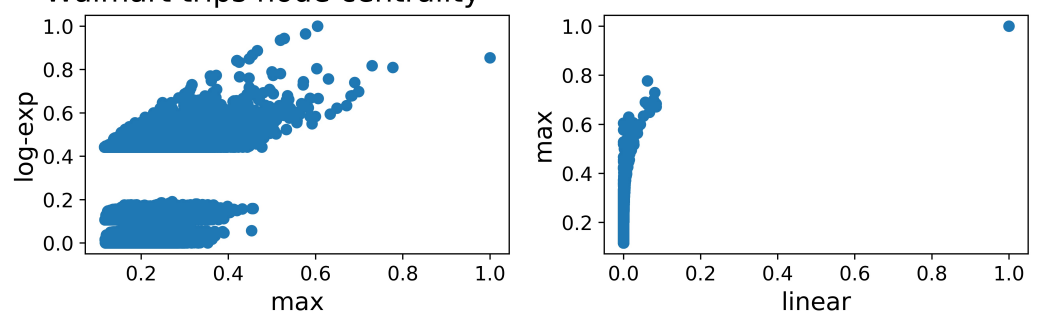

Math stackexchange co-trips edge centrality
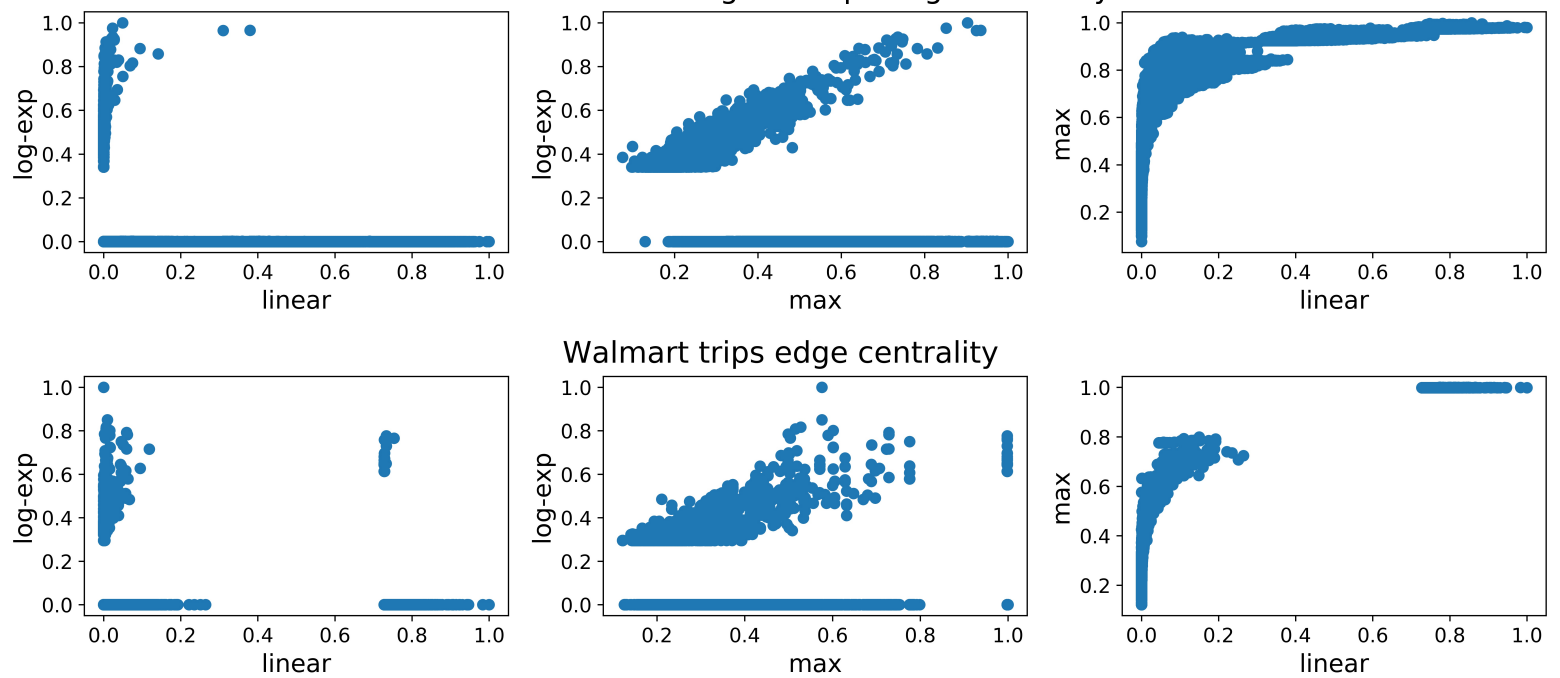

Figure 2: Scatter plots comparing node and edge centrality scores obtained with the singular vector model (2) for the three choices of the nonlinear functions discussed in Sec. 5.1.

between any pair of centralities, confirming that different choices of the nonlinear functions lead to remarkably different centrality score assignments.

As already observed for the case of the sunflower hypergraph, the 'linear' and 'log-exp' models may assign very different node centrality scores. This effect is also highlighted in Table 1 where we report the top ten nodes with highest centrality for the three models for the math stackexchange datasets. For this dataset nodes are the tags that posts receive on the math-stackexchange website.

A similar comparison is illustrated in Figure 3 where we scatter-plot the edge centrality of the three models with their edge weights. We see that, especially for the 'linear' and 'max' versions, larger edge weights do not correspond to greater importance in this spectral sense.

\section{Summary}

Centrality measures give useful information in a range of network science settings. In the study of on-line human behaviour, such measures are relevant to targeted advertising [25], and to the detection of fake news generation [28] and other negative behaviours such as the spread of viruses and cyberbullying [2]. 

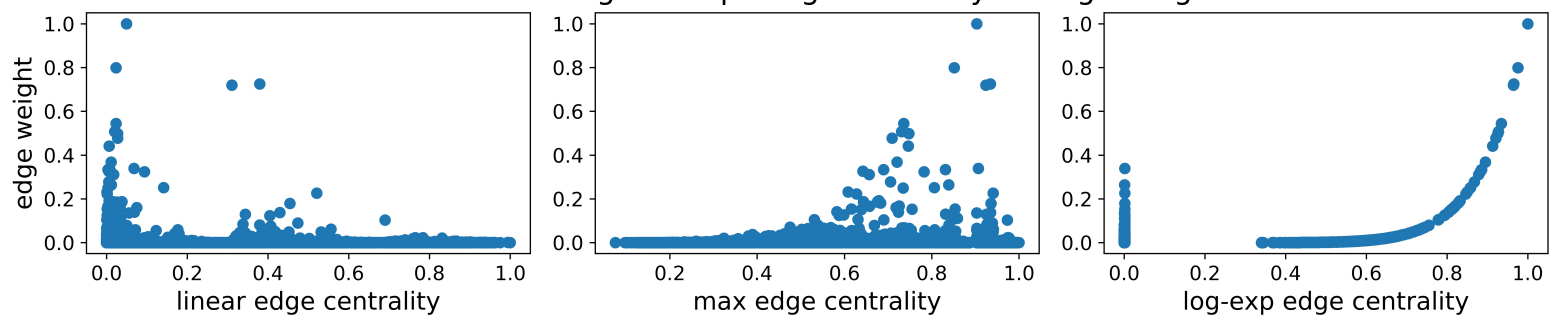

Walmart trips edge centrality vs edge weights

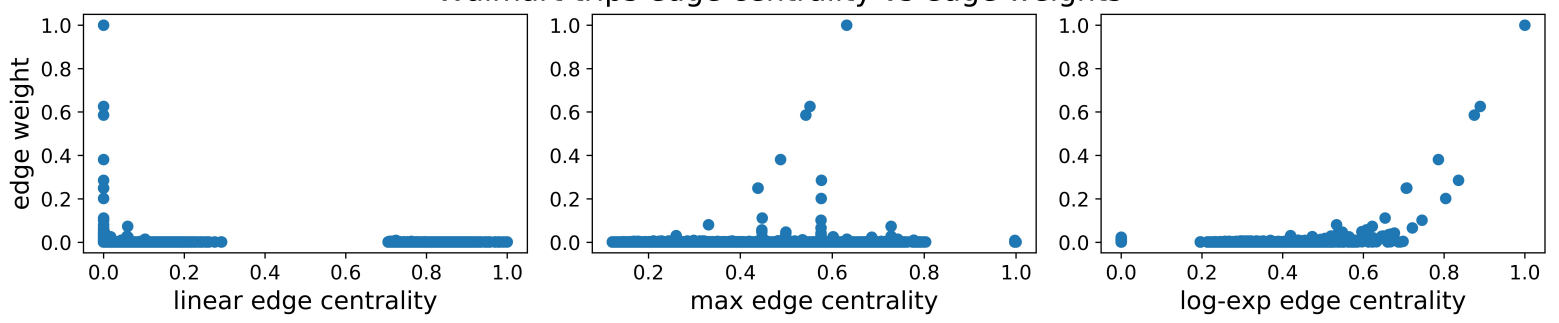

Figure 3: Scatter plot comparing the edge weights for both Walmart and Stackexchange datasets against the edge centrality score computed with the singular vector model (2) for the three choices of the nonlinear functions discussed in Sec. 5.1.

They have also proved useful in the physical world; for example in predicting (or vaccinating against) disease outbreaks [17], extracting biologically relevant features from neural connectivity data [14], and quantifying the attack robustness of power networks [12].

Taking the classical network view, where all nodes and pairwise connections play the same structural role, typically requires us to trade-off some fine detail for the sake of elegance and simplicity. By moving to higher order interactions, we are able to re-introduce some of this detail. Of course, in doing so we must understand the costs and benefits. Our aim in this work was to show that the widely-used spectral approach to centrality measurement can be extended rigorously and at very little cost to the general hypergraph setting in order to quantify the importance of both nodes and hyperedges. As shown in Theorem 4.1, there is a sound underlying theory behind the resulting constrained eigenvalue problems. Further, as shown in Theorem 4.2, the measures can be computed by an efficient and globally convergent iteration (Algorithm 1) that is built on matrix-vector products.

\section{Funding}

DJH was supported by EPSRC Programme Grant EP/P020720/1.

\section{Author contributions}

Both authors contributed to the formulation of the model, to the design of the algorithm and to its analysis. Moreover, the authors co-wrote the manuscript. FT wrote the code for the computational experiments. Both authors read and approved the final manuscript.

\section{Competing interests}

The authors declare that they have no competing financial interests

\section{Data availability}

All data and code used in this work are publicly available via the online repository https://github. com/ftudisco/node-edge-hypergraph-centrality 


\section{References}

[1] S. Agarwal, K. Branson, and S. Belongie. Higher order learning with graphs. In Proceedings of the 23rd International Conference on Machine Learning, pages 17-24, 2006.

[2] S. Ahajjam and H. Badir. Identification of influential spreaders in complex networks using HybridRank algorithm. Sci. Rep., 8, 2018.

[3] I. Amburg, N. Veldt, and A. R. Benson. Clustering in graphs and hypergraphs with categorical edge labels. In Proceedings of the Web Conference, 2020.

[4] F. Arrigo, D. J. Higham, and F. Tudisco. A framework for second-order eigenvector centralities and clustering coefficients. Proceedings of the Royal Society A, 476(2236):20190724, 2020.

[5] F. Arrigo and F. Tudisco. Multi-dimensional, multilayer, nonlinear and dynamic HITS. In Proceedings of the 2019 SIAM International Conference on Data Mining, pages 369-377. SIAM, 2019.

[6] F. Battiston, G. Cencetti, I. Iacopini, V. Latora, M. Lucas, A. Patania, J.-G. Young, and G. Petri. Networks beyond pairwise interactions: Structure and dynamics. Physics Reports, 874:1-92, 2020.

[7] A. R. Benson. Three hypergraph eigenvector centralities. SIAM Journal on Mathematics of Data Science, 1(2):293-312, 2019.

[8] A. R. Benson, R. Abebe, M. T. Schaub, A. Jadbabaie, and J. Kleinberg. Simplicial closure and higher-order link prediction. Proceedings of the National Academy of Sciences, 2018.

[9] P. Bonacich. Power and centrality: a family of measures. American Journal of Sociology, 92:1170$1182,1987$.

[10] A. Bretto. Hypergraph Theory: An introduction. Springer, Berlin, 2013.

[11] T. Bröhl and K. Lehnertz. Centrality-based identification of important edges in complex networks. Chaos, 29(3):033115, 2019.

[12] H. Cetinay, K. Devriendt, and P. V. Mieghem. Nodal vulnerability to targeted attacks in power grids. Applied Network Science, 3, 2018.

[13] S. Cipolla, M. Redivo-Zaglia, and F. Tudisco. Shifted and extrapolated power methods for tensor $\ell^{p}$-eigenpairs. ETNA: Electronic Transactions on Numerical Analysis, 53:1-27, 2020.

[14] J. J. Crofts and D. J. Higham. A weighted communicability measure applied to complex brain networks. Journal of the Royal Society Interface, 6:411-414.

[15] E. Estrada and P. A. Knight. A First Course in Network Theory. Oxford University Press, 2015.

[16] E. Estrada and J. A. Rodriguez-Velázquez. Subgraph centrality and clustering in complex hypernetworks. Physica A: Statistical Mechanics and its Applications, 364:581-594, 2006.

[17] M. Garcia-Herranz, E. Moro, M. Cebrian, N. A. Christakis, and J. H. Fowler. Using friends as sensors to detect global-scale contagious outbreaks. PLoS One, 9, 2014.

[18] A. Gautier and F. Tudisco. The contractivity of cone-preserving multilinear mappings. Nonlinearity, 32:4713, 2019.

[19] A. Gautier, F. Tudisco, and M. Hein. The Perron-Frobenius theorem for multihomogeneous mappings. SIAM Journal on Matrix Analysis and Applications, 40(3):1179-1205, 2019.

[20] A. Gautier, F. Tudisco, and M. Hein. A unifying Perron-Frobenius theorem for nonnegative tensors via multihomogeneous maps. SIAM Journal on Matrix Analysis and Applications, 40(3):12061231, 2019.

[21] D. Gleich and K. Kloster. Seeded pagerank solution paths. European Journal of Applied Mathematics, 27(6):812-845, 2016.

[22] Kaggle's Recruitment Prediction Competition. Walmart recruiting: Trip type classification. https : //www.kaggle.com/c/walmart-recruiting-trip-type-classification.

[23] M. Kim and J. Leskovec. The network completion problem: Inferring missing nodes and edges in networks. In Proceedings of the 2011 SIAM International Conference on Data Mining, 2012.

[24] J. M. Kleinberg. Authoritative sources in a hyperlinked environment. Journal of the ACM (JACM), 46(5):604-632, 1999. 
[25] P. Laflin, A. V. Mantzaris, P. Grindrod, F. Ainley, A. Otley, and D. J. Higham. Discovering and validating influence in a dynamic online social network. Social Network Analysis and Mining, 3:1311-1323, 2013.

[26] M. E. J. Newman. Networks: an Introduction. Oxford University Press, Oxford, 2010.

[27] X. Ouvrad. Hypergraphs: an introduction and review. Technical report, arXiv:2002.05014, 2010.

[28] F. Pierri, C. Piccardi, and S. Ceri. Topology comparison of Twitter diffusion networks effectively reveals misleading information. Sci. Rep., 10, 2020.

[29] J. P. Schäfermeyer. On Edmund Landau's contribution to the ranking of chess players. Technical report, Unpublished manuscript, 2019.

[30] D. Shin, S. Si, and I. S. Dhillon. Multi-scale link prediction. In Proceedings of the 21st ACM Conference on Information and Knowledge, 2012.

[31] L. Torres, A. S. Blevins, D. S. Bassett, and T. Eliassi-Rad. The why, how, and when of representations for complex systems. Technical report, arXiv:2006.02870v1, 2020.

[32] F. Tudisco, F. Arrigo, and A. Gautier. Node and layer eigenvector centralities for multiplex networks. SIAM Journal on Applied Mathematics, 78(2):853-876, 2018.

[33] F. Tudisco, V. Cardinali, and C. Di Fiore. On complex power nonnegative matrices. Linear Algebra and its Applications, 471:449-468, 2015.

[34] S. Vigna. Spectral ranking. Network Science, 4(4):433-445, 2016. 
Figures
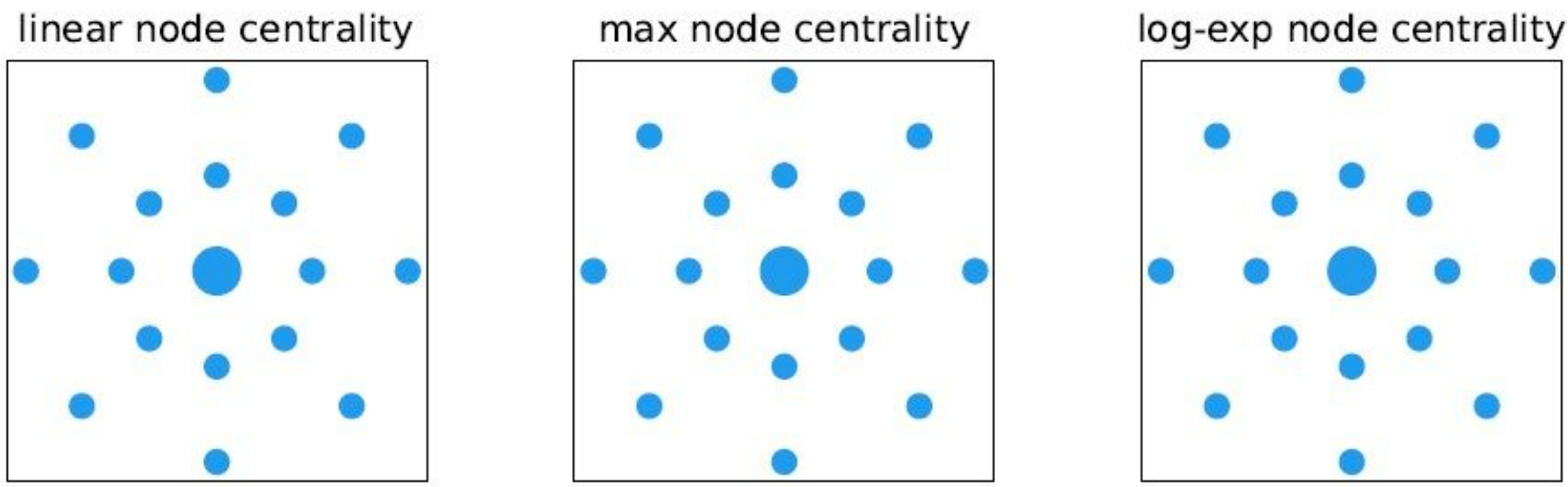

(a) Uniform sunflower with eight petals each containing three nodes

linear node centrality

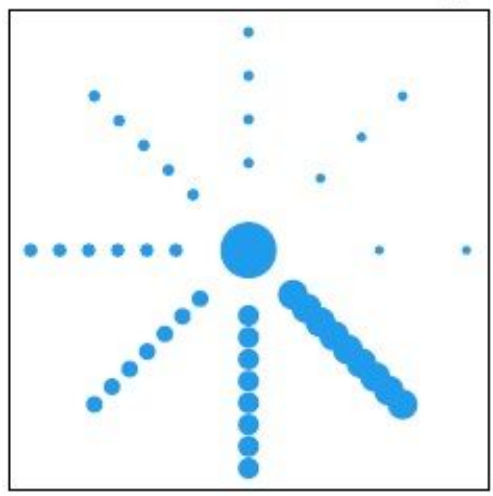

max node centrality

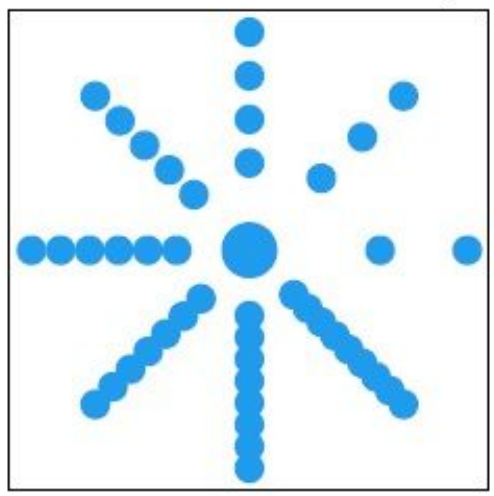

log-exp node centrality

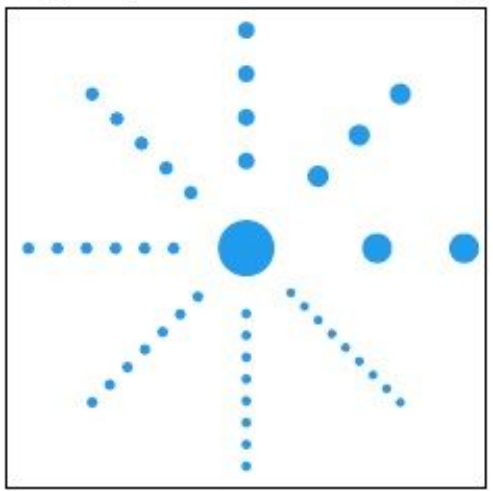

(b) General hypergraph sunflower with eight petals containing $3,4, \ldots, 10$ nodes, respectively.

\section{Figure 1}

Node centrality scores for the three models in Sec. 5.1 on the two example hypergraph sunflowers. Dots represent the hypergraph nodes and their size is proportional to their centrality value. 
Math stackexchange co-trips node centrality
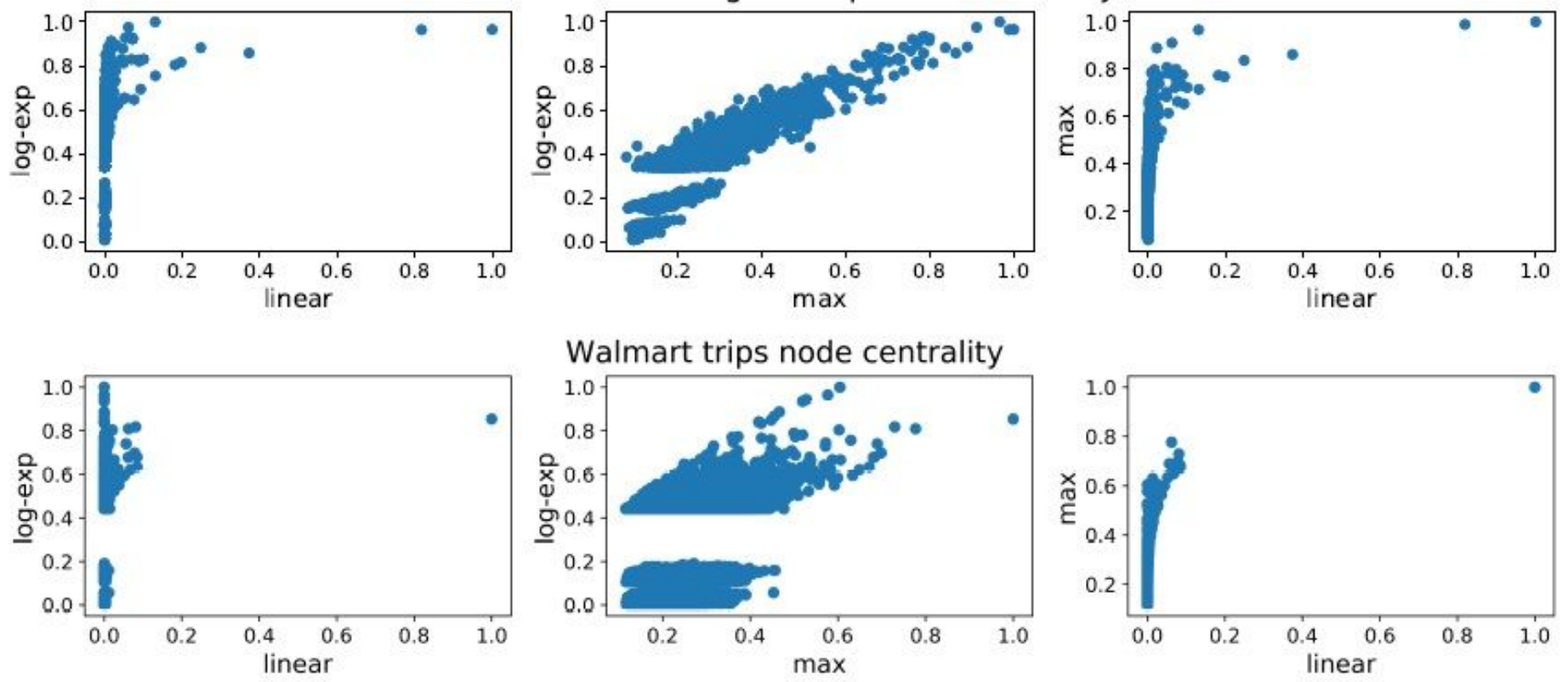

Walmart trips node centrality
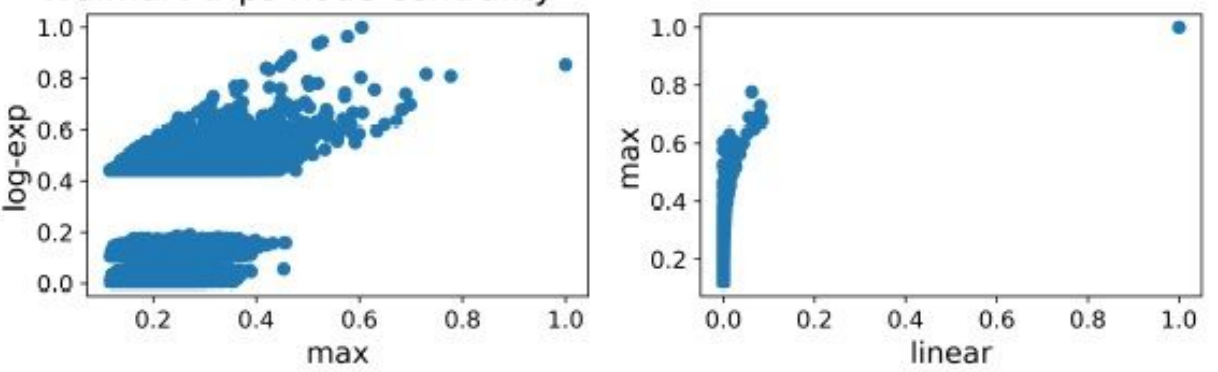

Math stackexchange co-trips edge centrality
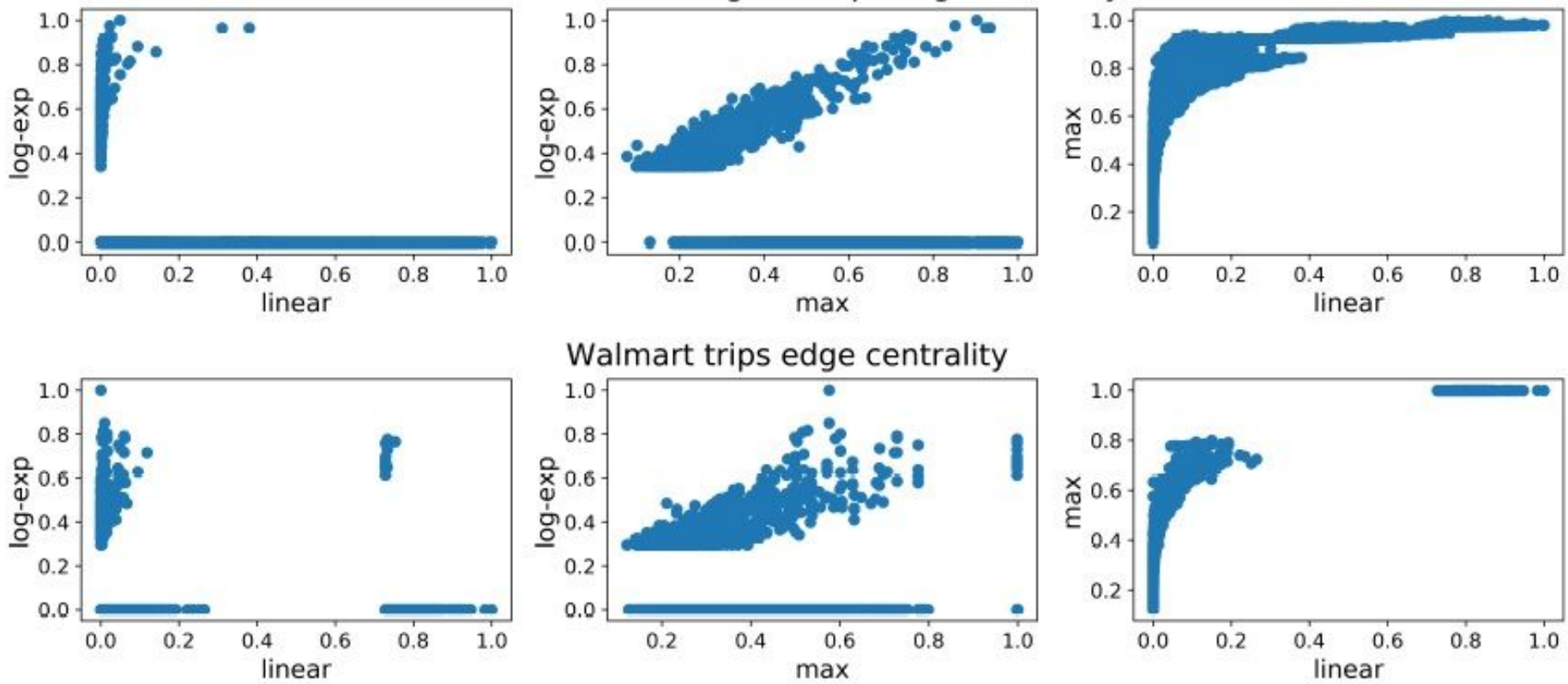

Walmart trips edge centrality
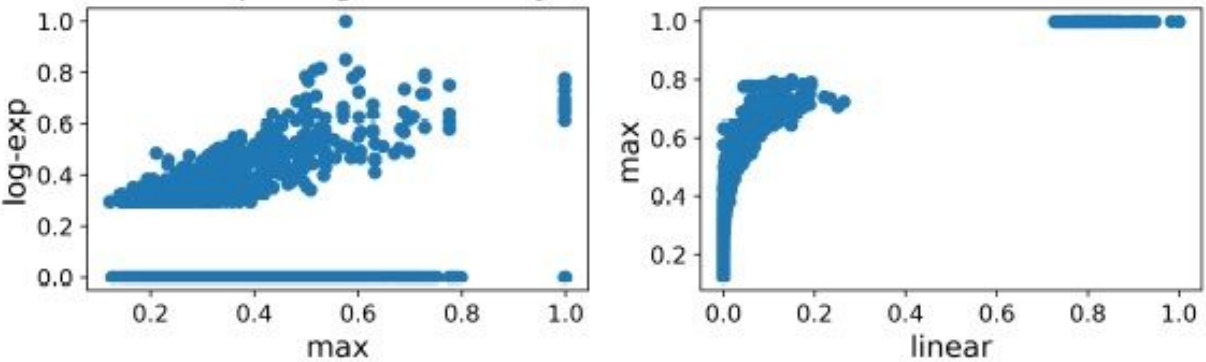

Figure 2

Scatter plots comparing node and edge centrality scores obtained with the singular vector model (2) for the three choices of the nonlinear functions discussed in Sec. 5.1. 
Math stackexchange co-trips edge centrality vs edge weights
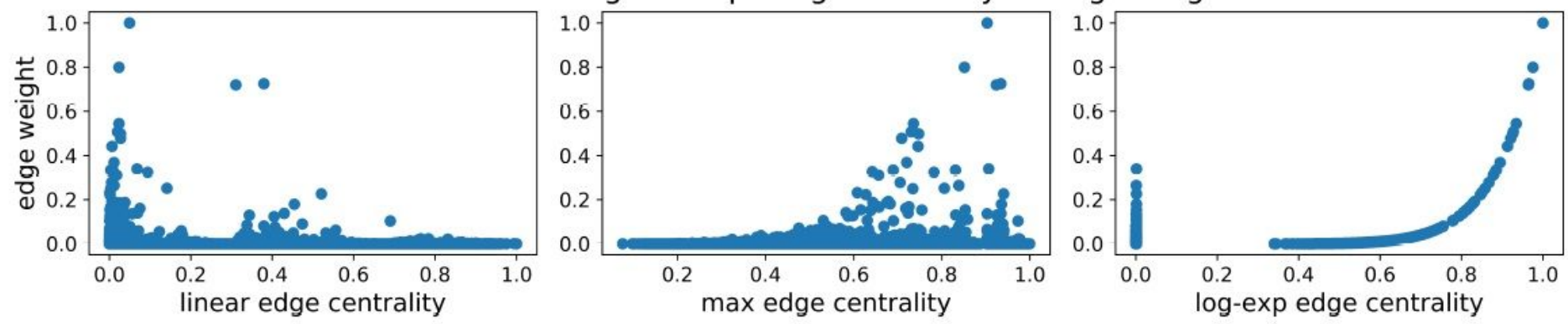

Walmart trips edge centrality vs edge weights
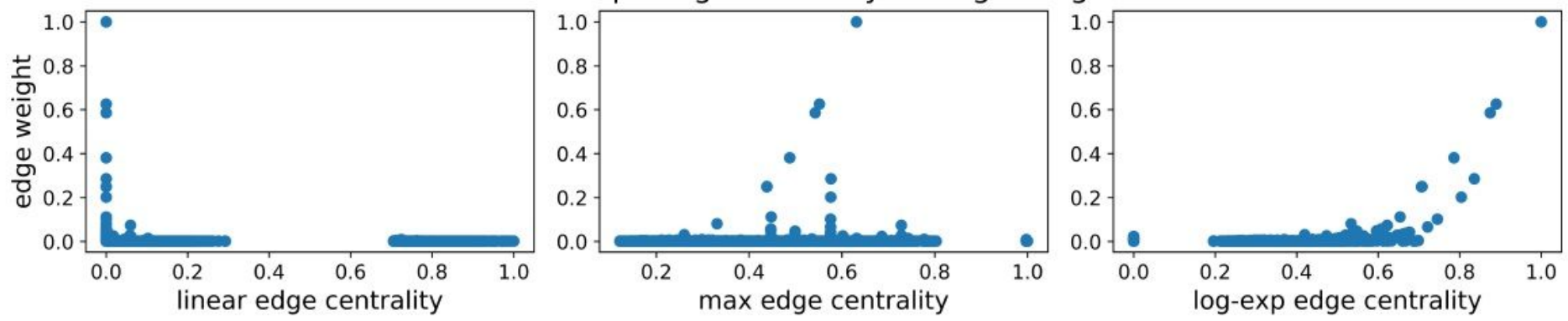

\section{Figure 3}

Scatter plot comparing the edge weights for both Walmart and Stackexchange datasets against the edge centrality score computed with the singular vector model (2) for the three choices of the nonlinear functions discussed in Sec. 5.1. 